\title{
SOFIA/EXES OBSERVATIONS OF WATER ABSORPTION IN THE PROTOSTAR AFGL 2591 AT HIGH SPECTRAL RESOLUTION
}

\author{
Nick Indriolo $^{1,9}$, D. A. Neufeld ${ }^{1}$, C. N. DeWitt ${ }^{2}$, M. J. Richter ${ }^{2}$, A. C. A. Boogert ${ }^{3}$, G. M. Harper ${ }^{4}$, \\ D. T. JAFFE ${ }^{5}$, K. R. Kulas ${ }^{6}$, M. E. McKelvey ${ }^{7}$, N. Ryde ${ }^{8}$, And W. VaCCA \\ ${ }^{1}$ Department of Physics and Astronomy, Johns Hopkins University, Baltimore, MD 21218, USA \\ ${ }^{2}$ Department of Physics, University of California Davis, Davis, CA 95616, USA \\ ${ }^{3}$ USRA, SOFIA, NASA Ames Research Center, MS 232-11, Moffett Field, CA 94035, USA \\ ${ }^{4}$ School of Physics, Trinity College, Dublin 2, Ireland \\ ${ }^{5}$ Department of Astronomy, University of Texas, Austin, TX 78712, USA \\ ${ }^{6}$ Department of Physics, Santa Clara University, Santa Clara, CA 95053, USA \\ ${ }^{7}$ NASA Ames Research Center, Moffett Field, CA 94035, USA \\ ${ }^{8}$ Department of Astronomy and Theoretical Physics, Lund University, Lund, Sweden \\ Received 2014 November 23; accepted 2015 February 20; published 2015 March 25
}

\begin{abstract}
We present high spectral resolution $\left(\sim 3 \mathrm{~km} \mathrm{~s}^{-1}\right)$ observations of the $\nu_{2}$ ro-vibrational band of $\mathrm{H}_{2} \mathrm{O}$ in the 6.086-6.135 $\mu \mathrm{m}$ range toward the massive protostar AFGL 2591 using the Echelon-Cross-Echelle Spectrograph (EXES) on the Stratospheric Observatory for Infrared Astronomy (SOFIA). Ten absorption features are detected in total, with seven caused by transitions in the $\nu_{2}$ band of $\mathrm{H}_{2} \mathrm{O}$, two by transitions in the first vibrationally excited $\nu_{2}$ band of $\mathrm{H}_{2} \mathrm{O}$, and one by a transition in the $\nu_{2}$ band of $\mathrm{H}_{2}^{18} \mathrm{O}$. Among the detected transitions is the $\nu_{2} 1_{1,1}-0_{0,0}$ line that probes the lowest-lying rotational level of para- $\mathrm{H}_{2} \mathrm{O}$. The stronger transitions appear to be optically thick, but reach maximum absorption at a depth of about $25 \%$, suggesting that the background source is only partially covered by the absorbing gas or that the absorption arises within the $6 \mu \mathrm{m}$ emitting photosphere. Assuming a covering fraction of $25 \%$, the $\mathrm{H}_{2} \mathrm{O}$ column density and rotational temperature that best fit the observed absorption lines are $N\left(\mathrm{H}_{2} \mathrm{O}\right)=(1.3 \pm 0.3) \times 10^{19} \mathrm{~cm}^{-2}$ and $T=640 \pm 80 \mathrm{~K}$.
\end{abstract}

Key words: stars: protostars

\section{INTRODUCTION}

Water, despite being one of the most abundant species in the molecular interstellar medium (ISM), is difficult to observe in astrophysical objects due to its prevalence in the Earth's atmosphere (see van Dishoeck et al. 2013 for a comprehensive review of astronomical water observations). Ground-based observations of $\mathrm{H}_{2} \mathrm{O}$ have primarily targeted maser emission, most frequently the $J_{K_{a} K_{c}}=6_{1,6}-5_{2,3}$ transition near $22 \mathrm{GHz}$ that was utilized in the initial detection of interstellar water (Cheung et al. 1969), although some have also focused on rotational and ro-vibrational transitions out of high-lying rotational levels in the mid-IR (Pontoppidan et al. 2010) and near-IR (Najita et al. 2000, p. 457; Carr et al. 2004; Salyk et al. 2008; Indriolo et al. 2013), respectively. These latter observations are possible because many high-lying rotational levels are not significantly populated in the Earth's atmosphere, but their scope is limited to astrophysical sources with warm $(T>300 \mathrm{~K})$, dense gas.

Observations of the lowest-lying rotational levels of waterthose able to probe cold gas-have required space-based observatories. The Infrared Space Observatory-Short Wavelength Spectrometer (ISO-SWS; de Graauw et al. 1996; Kessler et al. 1996) covered the $\nu_{2}$ ro-vibrational band (symmetric bending mode) of $\mathrm{H}_{2} \mathrm{O}$ centered near $6 \mu \mathrm{m}$, and absorption out of the lowest-lying levels of the ortho and para nuclear spin modifications $\left(1_{0,1}\right.$ and $0_{0,0}$, respectively) was detected toward several massive protostars (Boonman \& van Dishoeck 2003). Due to the low spectral resolution of the observing configuration though $(\lambda / \Delta \lambda \sim 1400$ using SWS in AOT6 grating

\footnotetext{
9 Current address: Department of Astronomy, University of Michigan, Ann Arbor, MI 48109, USA
}

mode), these lines were significantly blended with absorption from other nearby $\mathrm{H}_{2} \mathrm{O}$ lines, making the determination of level-specific column densities impossible. Instead, the entire $\nu_{2}$ band was fit simultaneously assuming a single temperature to determine the total water column density, $N\left(\mathrm{H}_{2} \mathrm{O}\right)$. The Submillimeter Wave Astronomy Satellite (SWAS; Melnick et al. 2000b) provided much higher spectral resolution $(\lesssim 1$ $\mathrm{km} \mathrm{s}^{-1}$ ) and covered the $1_{1,0}-1_{0,1}$ pure rotational transition of $\mathrm{H}_{2} \mathrm{O}$ at $557 \mathrm{GHz}$. This line was observed in both emission and absorption in multiple sources (e.g., Melnick et al. 2000a; Snell et al. 2000), demonstrating the ability to probe cold water. More recently, the study of low-lying rotational levels at high spectral resolution $\left(\sim 0.5 \mathrm{~km} \mathrm{~s}^{-1}\right)$ has been facilitated by the Heterodyne Instrument for the Far-Infrared (HIFI; de Graauw et al. 2010) on board the Herschel Space Observatory (Pilbratt et al. 2010). Water has been detected in both emission and absorption out of levels with $E \lesssim 200 \mathrm{~K}$ in several protostars (e.g., van Dishoeck et al. 2011; van der Tak et al. 2013), and in absorption out of the $0_{0,0}$ and $1_{0,1}$ levels in the molecular ISM (e.g., Sonnentrucker et al. 2010; Flagey et al. 2013). Observations that resolve the velocity structure of absorption lines are vital to both determining level-specific column densities and understanding the dynamics of the absorbing/emitting regions. This is especially important for protostars as such objects contain multiple dynamical components (e.g., disk, envelope, jets, outflows, shocks).

AFGL 2591 is a region of ongoing high-mass star formation. The radio continuum source VLA 3 (for a description of sources, see Torrelles et al. 2014 and references therein) is the brightest mid-IR source in the region and drives a bipolar outflow. It was previously observed at $5-7 \mu \mathrm{m}$ as part of the aforementioned ISO-SWS study, from which Boonman \& van 


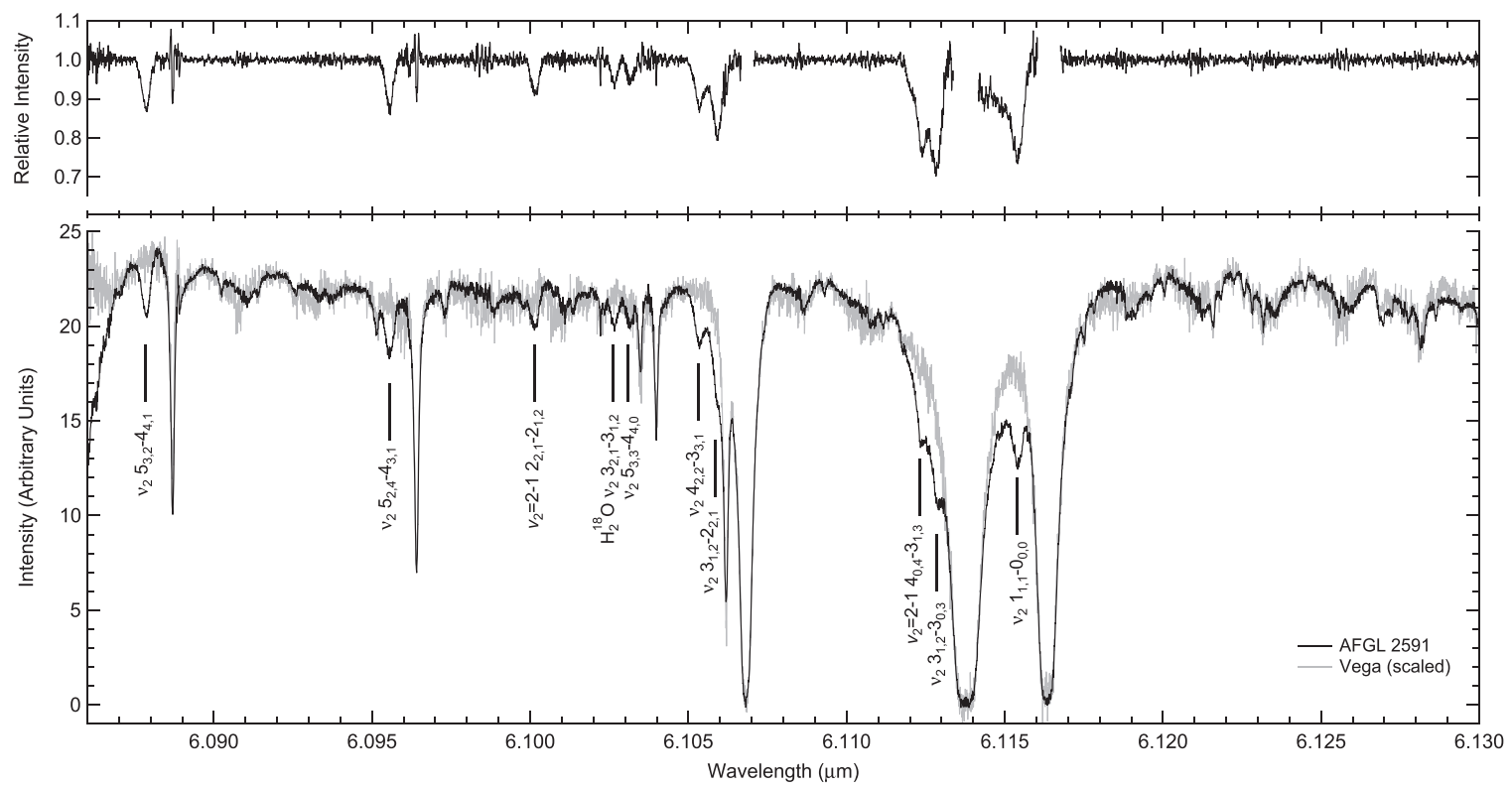

Figure 1. Bottom: spectra of AFGL 2591 (black) and Vega (gray) scaled to match in arbitrary intensity units. Water absorption features in AFGL 2591are marked by vertical lines and labeled accordingly. They are blueshifted by about $40 \mathrm{~km} \mathrm{~s}^{-1}$ with respect to their telluric counterparts due to the Earth's motion and gas velocity. Top: spectrum of AFGL 2591 following division by boxcar averaged Vega spectrum and normalization. Gaps correspond to regions where the atmosphere is opaque, and poor removal of moderately strong atmospheric lines can be seen as spikes to the right of the astrophysical lines (e.g., near $6.089 \mu \mathrm{m})$.

Dishoeck (2003) reported a best-fit water column density of $N\left(\mathrm{H}_{2} \mathrm{O}\right)=(3.5 \pm 1.5) \times 10^{18} \mathrm{~cm}^{-2} \quad$ at $T=450_{-150}^{+250} \mathrm{~K}$, assuming a Doppler line width of $5 \mathrm{~km} \mathrm{~s}^{-1}$. Known kinematic components associated with AFGL 2591 include (1) the protostellar envelope with systemic velocity $-5.5 \mathrm{~km} \mathrm{~s}^{-1}$ in the local standard of rest (LSR) frame (van der Tak et al. 1999); (2) a blueshifted outflow at $-25 \mathrm{~km}$ $\mathrm{s}^{-1} \lesssim v_{\mathrm{LSR}} \lesssim-6 \mathrm{~km} \mathrm{~s}^{-1}$ (Emprechtinger et al. 2012; van der Tak et al. 2013); (3) a redshifted outflow at $3 \mathrm{~km}$ $\mathrm{s}^{-1} \lesssim v_{\mathrm{LSR}} \lesssim 15 \mathrm{~km} \mathrm{~s}^{-1}$ (Lada et al. 1984); (4) material entrained in a jet or wind, indicated by absorption in the $v=1-0$ band of ${ }^{12} \mathrm{CO}$ that extends to $-196 \mathrm{~km} \mathrm{~s}^{-1}$ in the form of an asymmetric blue wing (van der Tak et al. 1999). There is also evidence of a rotating disk around VLA 3, although gas velocities in the disk match those of the envelope (van der Tak et al. 2006; Wang et al. 2012). The interested reader is referred to cartoon pictures (van der Tak et al. 1999, 2006; van der Wiel et al. 2011; Sanna et al. 2012; Wang et al. 2012) and multi-wavelength images (Johnston et al. 2013) of AFGL 2591 to gain a better understanding of the region. Because of the low spectral resolution of the ISO-SWS observations, it has been impossible to say with certainty which component gives rise to the $\mathrm{H}_{2} \mathrm{O}$ absorption. To do so- and to better constrain the water column density and rotational temperature-we have targeted multiple transitions in the $\nu_{2}$ band of $\mathrm{H}_{2} \mathrm{O}$ using the Echelon-CrossEchelle Spectrograph (EXES; Richter et al. 2010) on board the Stratospheric Observatory for Infrared Astronomy (SOFIA; Young et al. 2012).

SOFIA operates at altitudes above $39,000 \mathrm{ft}(11,887 \mathrm{~m})$, where the precipitable water vapor overburden is routinely less than $0.02 \mathrm{~mm}$. Under these conditions the $6 \mu \mathrm{m}$ region of the Earth's atmosphere is no longer opaque, as it is from the ground. EXES provides high spectral resolution $\left(\sim 3 \mathrm{~km} \mathrm{~s}^{-1}\right)$ capabilities in the $4.5-28.3 \mu \mathrm{m}$ range, making it well-suited for velocity-resolved observations of individual ro-vibrational transitions of the $\nu_{2}$ band of $\mathrm{H}_{2} \mathrm{O}$ in astrophysical sources. We present here the first spectrally resolved detections of 10 absorption lines from transitions in the $\nu_{2}$ bands of $\mathrm{H}_{2} \mathrm{O}$ and $\mathrm{H}_{2}^{18} \mathrm{O}$, including a detection probing the ground para level, $0_{0,0}$. This highlights the opportunity to further probe water in cold molecular clouds without the need for a space-based observatory by utilizing EXES on SOFIA.

\section{OBSERVATIONS AND DATA REDUCTION}

AFGL 2591 VLA 3 was observed using EXES on board SOFIA at an altitude of $43,000 \mathrm{ft}(13,106 \mathrm{~m})$ on 2014 April 10 (UT) as part of instrument commissioning observations. Spectra were acquired in cross-dispersed high-resolution mode with a central wavelength of $6.1125 \mu \mathrm{m}$, using a slit length of $9 ! .9$, and a slit width of 1.9 to provide a resolving power (resolution) of $86,000\left(3.5 \mathrm{~km} \mathrm{~s}^{-1}\right)$, with the resolution element sampled by 8 pixels. The telescope was nodded after every $27 \mathrm{~s}$ of integration, enabling subtraction of telluric emission lines, and the total exposure time for AFGL 2591 was 1134 s. Prior to observing the target, a calibration sequence was taken using the same wavelength setting and slit width. This sequence consisted of observations of the internal blackbody unit set to $260 \pm 0.1 \mathrm{~K}$ and of blank sky (Lacy et al. 2002). These calibration frames were used to correct for blaze efficiency, pixel-to-pixel sensitivity variations, and also to provide a first order flux calibration using the expected photon counts from the blackbody unit. The bright star Vega was observed during a flight leg $2 \mathrm{hr}$ before the science observations at the same altitude and air mass as AFGL 2591 for use as a telluric standard star. The observing sequence was similar to that employed for AFGL 2591.

Data were processed using the Redux pipeline (Clarke et al. 2015) with the fspextool software package-a modification of the Spextool package (Cushing et al. 2004) -which performs source profile construction, extraction and background aperture definition, optimal extraction, and wavelength calibration for EXES data. The preliminary wavelength scale output from the pipeline was refined by shifting the 


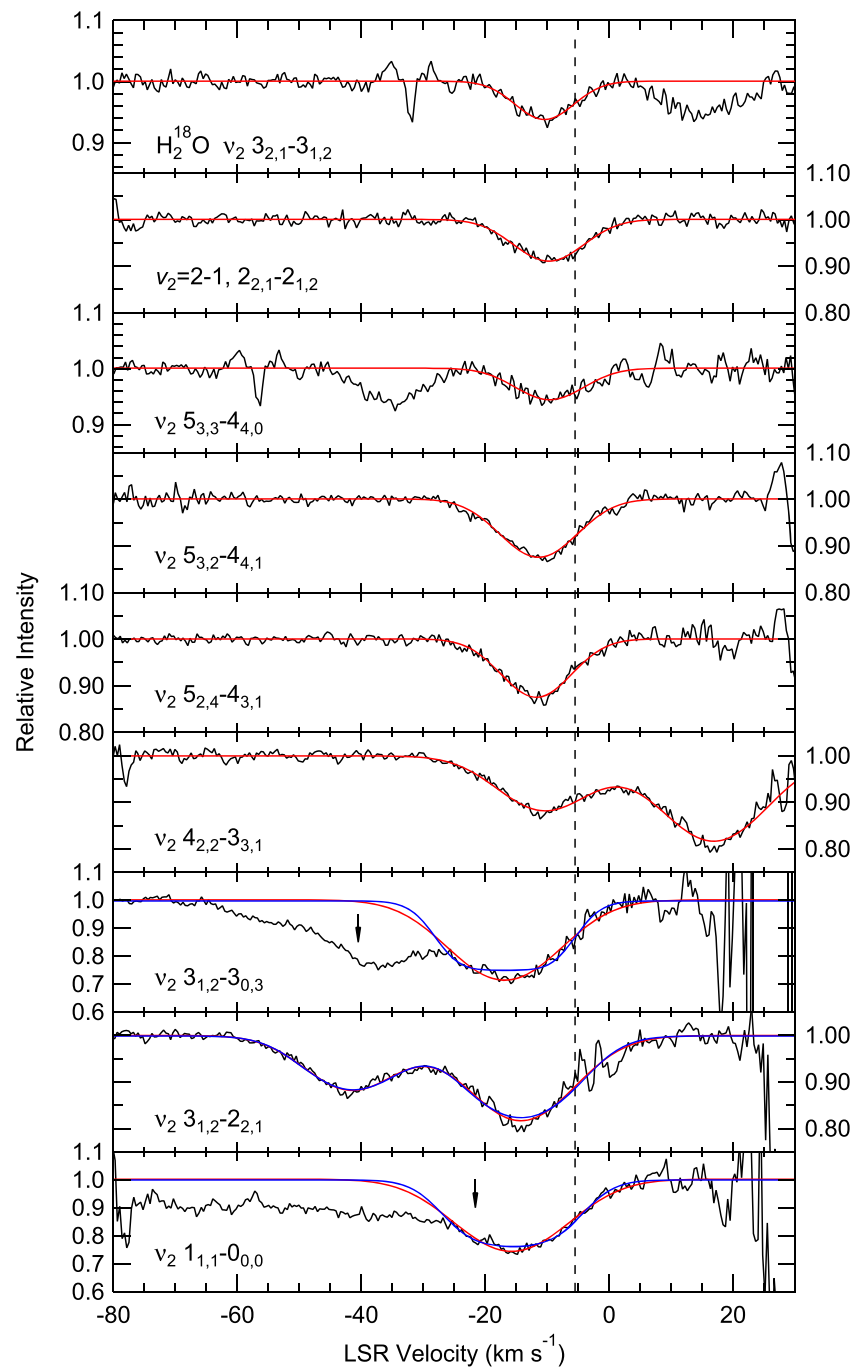

Figure 2. Individual $\mathrm{H}_{2} \mathrm{O}$ absorption lines as functions of LSR velocity, arranged in order of increasing lower state energy (except the $\mathrm{H}_{2}^{18} \mathrm{O}$ line). The vertical dashed line shows the systemic velocity of AFGL 2591 determined from various molecular emission lines $\left(v_{\mathrm{LSR}}=-5.5 \mathrm{~km} \mathrm{~s}^{-1}\right.$; van der Tak et al. 1999). Red and blue curves show fits with $f_{c}=1$ and $f_{c}=0.25$, respectively. For optically thin lines both fits are nearly identical, so only the $f_{c}=1$ fits are shown. At $v_{\mathrm{LSR}} \lesssim-25 \mathrm{~km} \mathrm{~s}^{-1}$ in the $v_{2} 1_{1,1}-0_{0,0}$ spectrum we consider the lower continuum level to be caused by poor removal of strong atmospheric lines. The arrow at $-22 \mathrm{~km} \mathrm{~s}^{-1}$ marks the expected position of the $v_{2}=2-13_{1,3}-2_{0,2}$ transition given a line center of $-11 \mathrm{~km} \mathrm{~s}^{-1}$. In the $\nu_{2} 3_{1,2}-3_{0,3}$ spectrum the arrow at $-41 \mathrm{~km} \mathrm{~s}^{-1}$ marks the expected position of the $v_{2}=2-14_{0,4}-3_{1,3}$ transition, and we consider the feature from about $-32 \mathrm{~km} \mathrm{~s}^{-1}$ to $-47 \mathrm{~km} \mathrm{~s}^{-1}$ to be a detection of this line, although it is also affected by a sloping baseline due to poor removal of atmospheric lines.

wavelength solution so that telluric water absorption lines in the AFGL 2591 continuum best matched entries in the HITRAN database (Rothman et al. 2013). This wavelength calibration is accurate to within $\pm 0.3 \mathrm{~km} \mathrm{~s}^{-1}$. Spectra extracted from individual orders of the echellogram were combined to form a single continuous spectrum in the wavelength range 6.085-6.135 $\mu \mathrm{m}$ for both AFGL 2591 and Vega as shown in the bottom panel of Figure 1. To remove atmospheric features, the spectrum of AFGL 2591 was divided by a smoothed (11 point boxcar average) Vega spectrum. The resulting ratioed spectrum was then divided by a boxcar average to the continuum level extrapolated across absorption lines to produce the normalized spectrum in the top panel of Figure 1. Figure 2 shows the spectra for each transition shifted into the LSR frame following conversion from wavelength to line-of-sight velocity.

\section{ANALYSIS}

Absorption lines in Figure 2 were fit using a function of the form

$$
I=I_{0}\left[1-f_{c}\left[1-\exp \left(-\tau_{0} \exp \left(-\frac{\left(v-v_{\mathrm{LSR}}\right)^{2}}{2 \sigma_{v}^{2}}\right)\right)\right]\right],
$$

where $I_{0}$ is the continuum level, $f_{c}$ is the fraction of the background source covered by absorbing material, and the optical depth is assumed to have a Gaussian profile with optical depth at line center $\tau_{0}$, LSR velocity at line center $v_{\mathrm{LSR}}$, and velocity dispersion $\sigma_{v}$. The $\nu_{2} \quad 3_{1,2}-2_{2,1}$ and $\nu_{2} 4_{2,2}-3_{3,1}$ transitions were fit simultaneously (second and fourth panels from the bottom in Figure 2) using a version of this function modified for multiple lines. The $\nu_{2} 5_{3,3}-4_{4,0}$ and $\mathrm{H}_{2}^{18} \mathrm{O} \nu_{2} 3_{2,1^{-}}$ $3_{1,2}$ transitions (third from top and top panels) were fit separately despite their proximity to each other as there is no significant blending of the features. Due to interfering features near the $\nu_{2} 1_{1,1}-0_{0,0}$ and $\nu_{2} 3_{1,2}-3_{0,3}$ transitions (bottom and third from bottom panels; see figure caption for explanation of features) only data at $v_{\mathrm{LSR}} \geqslant-23 \mathrm{~km} \mathrm{~s}^{-1}$ were used to constrain fits of these transitions. As the absorption features are broad with respect to the instrumental spectral resolution, we expect the lines to be resolved and convert the optical depth profile fit to a column density profile via

$$
d N / d v=\tau(v) \frac{g_{l}}{g_{u}} \frac{8 \pi}{A \lambda^{3}},
$$

under the assumption that the absorption is unsaturated, where $g_{l}$ and $g_{u}$ are statistical weights in the lower and upper states, respectively, $A$ is the spontaneous emission coefficient, and $\lambda$ is the transition wavelength. We then integrate over the absorption feature in velocity space to determine the column density in the lower state of the observed transition.

Initial fits were made assuming that the absorbing gas completely covers the background source $\left(f_{c}=1\right)$ and are shown as red curves in Figure 2. Resulting parameters for each transition are reported in Table 1. Inferred column densities are converted to $\ln \left(f_{c} N / g_{l}\right)$ and plotted versus lower state energy as red squares in Figure 3. The black dashed line shows the relationship expected for $N\left(\mathrm{H}_{2} \mathrm{O}\right)=3.5 \times 10^{18} \mathrm{~cm}^{-2}$ in local thermodynamic equilibrium (LTE) at $T=450 \mathrm{~K}$ (Boonman \& van Dishoeck 2003). Column densities in the $3_{3,1}, 4_{3,1}, 4_{4,1}$, and $4_{4,0}$ states are in agreement with predictions based on these values, but the states with $E / k_{B}<300 \mathrm{~K}$ are below predicted values, while vibrationally excited states are above predicted values. No single temperature provides a good fit to all nine points, but if we exclude levels with $E / k_{B}<300 \mathrm{~K}$ then we find $N\left(\mathrm{H}_{2} \mathrm{O}\right)=(3.7 \pm 0.8) \times 10^{18} \mathrm{~cm}^{-2}$ in LTE at $T=590 \pm 50 \mathrm{~K}$ (marked by the red dotted line in Figure 3).

Assuming $T$ and $N\left(\mathrm{H}_{2} \mathrm{O}\right)$ from Boonman \& van Dishoeck (2003), the $\nu_{2} 3_{1,2}-3_{0,3}$ transition should be saturated at line center, which is not the case. If the background source is partially covered by absorbing material or if the water absorption arises within the $6 \mu \mathrm{m}$ emitting photosphere, then it is possible for optically thick, saturated lines to cause only a 
Table 1

Observed Water Transitions and Inferred Parameters

\begin{tabular}{|c|c|c|c|c|c|c|c|c|c|}
\hline & Transition $^{\mathrm{a}}$ & $\begin{array}{l}\text { Wavelength } \\
\quad(\mu \mathrm{m})\end{array}$ & $\begin{array}{c}E_{l} / k_{b} \\
(\mathrm{~K})\end{array}$ & $g_{l}$ & $\begin{array}{c}A \\
\left(\mathrm{~s}^{-1}\right)\end{array}$ & $\begin{array}{c}v_{\mathrm{LSR}} \\
\left(\mathrm{km} \mathrm{s}^{-1}\right)\end{array}$ & $\begin{array}{c}\sigma_{v} \\
\left(\mathrm{~km} \mathrm{~s}^{-1}\right)\end{array}$ & $\tau_{0}$ & $\ln \left(f_{c} N_{l} / g_{l}\right)$ \\
\hline \multicolumn{10}{|c|}{ Fit Results for $f_{c}=1$} \\
\hline \multirow{10}{*}{$\mathrm{H}_{2}^{18} \mathrm{O}$} & $1-05_{3,2}-4_{4,1}$ & 6.0887005 & 702.3 & 27 & 0.35 & -11.4 & 6.0 & $0.13 \pm 0.03$ & $35.2 \pm 0.3$ \\
\hline & $1-05_{2,4}-4_{3,1}$ & 6.0964081 & 552.3 & 9 & 0.73 & -11.7 & 5.5 & $0.13 \pm 0.03$ & $35.5 \pm 0.3$ \\
\hline & $2-12_{2,1}-2_{1,2}$ & 6.1009690 & 2412.9 & 15 & 5.92 & -9.7 & 5.5 & $0.09 \pm 0.03$ & $32.7 \pm 0.4$ \\
\hline & $1-03_{2,1}-3_{1,2}$ & 6.1034870 & 248.7 & 21 & 6.40 & -10.4 & 4.7 & $0.06 \pm 0.03$ & $31.8 \pm 0.5$ \\
\hline & $1-05_{3,3}-4_{4,0}$ & 6.1039868 & 702.3 & 9 & 0.34 & -9.6 & 5.5 & $0.06 \pm 0.03$ & $35.4 \pm 0.6$ \\
\hline & $1-04_{2,2}-3_{3,1}$ & 6.1061925 & 410.4 & 7 & 0.53 & -10.5 & 7.3 & $0.12 \pm 0.03$ & $36.2 \pm 0.3$ \\
\hline & $1-03_{1,2}-2_{2,1}$ & 6.1068262 & 194.1 & 15 & 1.12 & -14.2 & 8.5 & $0.20 \pm 0.04$ & $35.2 \pm 0.2$ \\
\hline & $2-14_{0,4}-3_{1,3}$ & 6.1131638 & 2502.7 & 7 & 15.8 & -8.4 & 4.4 & $0.12 \pm 0.03$ & $32.2 \pm 0.3$ \\
\hline & $1-03_{1,2}-3_{0,3}$ & 6.1137707 & 196.8 & 21 & 6.24 & -16.9 & 8.5 & $0.34 \pm 0.04$ & $34.0 \pm 0.1$ \\
\hline & $1-01_{1,1}-0_{0,0}$ & 6.1163311 & 0 & 1 & 7.46 & -15.7 & 8.7 & $0.30 \pm 0.04$ & $35.7 \pm 0.1$ \\
\hline \multicolumn{10}{|c|}{ Fit Results for $f_{c}=0.25$} \\
\hline \multirow{10}{*}{$\mathrm{H}_{2}^{18} \mathrm{O}$} & $1-05_{3,2}-4_{4,1}$ & 6.0887005 & 702.3 & 27 & 0.35 & -11.4 & 5.6 & $0.68_{-0.21}^{+0.27}$ & $35.4_{-0.3}^{+0.4}$ \\
\hline & $1-05_{2,4}-4_{3,1}$ & 6.0964081 & 552.3 & 9 & 0.73 & -11.8 & 5.2 & $0.68_{-0.21}^{+0.27}$ & $35.6_{-0.3}^{+0.4}$ \\
\hline & $2-12_{2,1}-2_{1,2}$ & 6.1009690 & 2412.9 & 15 & 5.92 & -9.8 & 5.3 & $0.44_{-0.18}^{+0.21}$ & $32.8_{-0.4}^{+0.5}$ \\
\hline & $1-03_{2,1}-3_{1,2}$ & 6.1034870 & 248.7 & 21 & 6.40 & -10.4 & 4.5 & $0.29_{-0.15}^{+0.17}$ & $31.8_{-0.5}^{+0.6}$ \\
\hline & $1-05_{3,3}-4_{4,0}$ & 6.1039868 & 702.3 & 9 & 0.34 & -9.6 & 5.3 & $0.25_{-0.14}^{+0.17}$ & $35.4_{-0.6}^{+0.7}$ \\
\hline & $1-04_{2,2}-3_{3,1}$ & 6.1061925 & 410.4 & 7 & 0.53 & -10.3 & 6.9 & $0.62_{-0.20}^{+0.25}$ & $36.4_{-0.3}^{+0.4}$ \\
\hline & $1-03_{1,2}-2_{2,1}$ & 6.1068262 & 194.1 & 15 & 1.12 & -14.2 & 7.3 & $1.20_{-0.34}^{+0.51}$ & $35.5_{-0.3}^{+0.4}$ \\
\hline & $2-14_{0,4}-3_{1,3}$ & 6.1131638 & 2502.7 & 7 & 15.8 & -8.4 & 4.1 & $0.57_{-0.19}^{+0.24}$ & $32.4_{-0.3}^{+0.4}$ \\
\hline & $1-03_{1,2}-3_{0,3}$ & 6.1137707 & 196.8 & 21 & 6.24 & -16.8 & 5.7 & $>5.1$ & $>35.0$ \\
\hline & $1-01_{1,1}-0_{0,0}$ & 6.1163311 & 0 & 1 & 7.46 & -15.6 & 6.5 & $>3.0$ & $>36.3$ \\
\hline
\end{tabular}

Notes. Columns 2-5 give the transition wavelength, $\lambda$, lower state energy, $E_{l} / k_{b}$, lower-state statistical weight, $g_{l}$, and the spontaneous emission coefficient, $A$, respectively. Columns 6-8 give the best-fit parameters from Equation (1), with covering fractions of $100 \%$ and $25 \%$ assumed in the top and bottom halves of the table, respectively. Uncertainties in $v_{\mathrm{LSR}}$ and $\sigma_{v}$ are estimated to be $0.3 \mathrm{~km} \mathrm{~s}^{-1}$ at the $1 \sigma$ level. Uncertainties in $\tau_{0}$ assume that the uncertainty in the absorption depth at line center is equal to the root mean square noise level of 0.03 in the continuum of the ratioed spectrum prior to the removal of baseline fluctuations. The Doppler parameter, $b$, and line FWHM are related to $\sigma_{v}$ via the equations $b=\sigma_{v} \sqrt{2}$ and FWHM $=\sigma_{v} 2 \sqrt{2 \ln (2)}$.

${ }^{\text {a }}$ Transition labels are given as $v_{2}^{\prime}-v_{2}^{\prime \prime} J_{K_{a}^{\prime} K_{c}^{\prime}}^{\prime}-J_{K_{a}^{\prime \prime} K_{c}^{\prime \prime}}^{\prime \prime}$, where a single prime denotes the upper state and double prime denotes the lower state, and both the $v_{1}$ and $v_{3}$ vibrational quantum numbers are omitted as they are 0 in all cases.

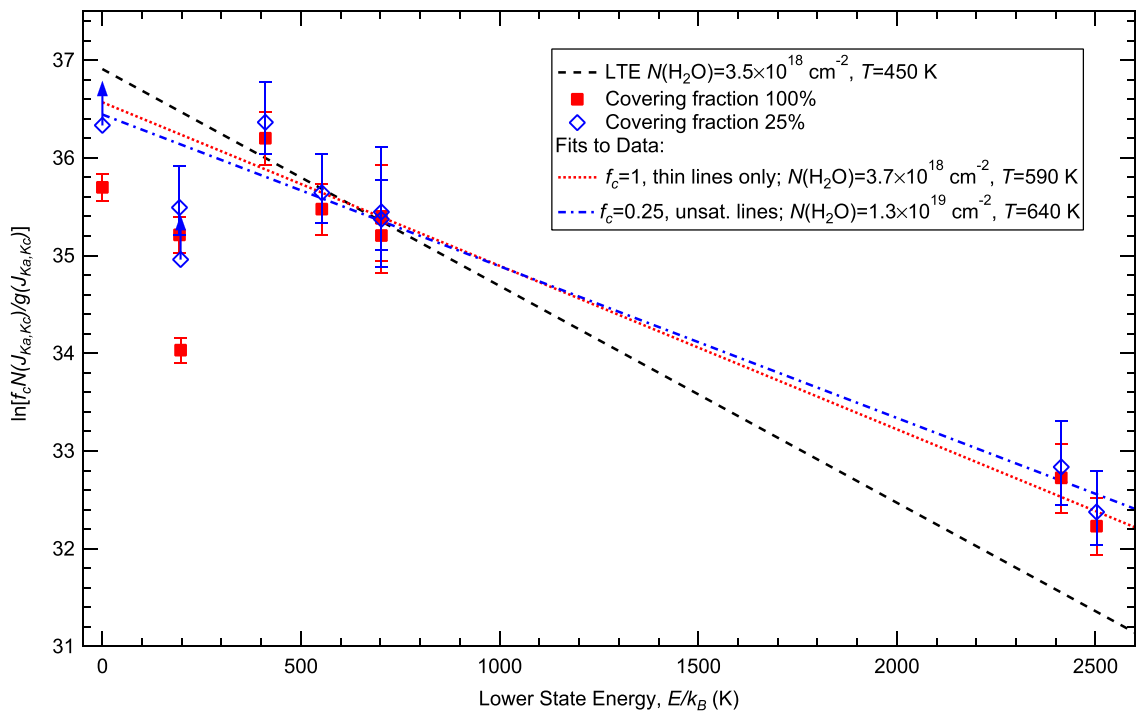

Figure 3. Rotation diagram for the nine detected $\mathrm{H}_{2} \mathrm{O}$ transitions. The black dashed line is for $N\left(\mathrm{H}_{2} \mathrm{O}\right)=3.5 \times 10^{18} \mathrm{~cm}^{-2}$ and LTE at $450 \mathrm{~K}$ as reported by Boonman $\&$ van Dishoeck (2003). Red squares and open blue diamonds denote covering fractions of 1 and 0.25 , respectively. Lower limits are indicated by upward-pointing arrows. Note that the $y$-axis shows $\ln \left(f_{c} N\left(J_{K_{a} K_{c}}\right) / g_{K_{a} K_{c}}\right)$, (i.e., is scaled to the average column density in front of the entire background source). For optically thin absorption lines the value does not change significantly with covering fraction, but for optically thick lines (e.g., $\left.\nu_{2} 1_{1,1}-0_{0,0}\right)$ the value increases substantially as $f_{c}$ approaches the absorption depth. The red dotted line marks $T=590 \mathrm{~K}$ and $N\left(\mathrm{H}_{2} \mathrm{O}\right)=3.7 \times 10^{18} \mathrm{~cm}^{-2}$ for $f_{c}=1$, and is the best fit to levels with $E / k_{B}>300 \mathrm{~K}$. The blue dash-dot line marks $T=640 \mathrm{~K}$ and $N\left(\mathrm{H}_{2} \mathrm{O}\right)=1.3 \times 10^{19} \mathrm{~cm}^{-2}$ for $f_{c}=0.25$, and is the best fit to the seven unsaturated transitions. 
fractional decrease in the continuum level. The minimum possible fractional coverage is equal to the depth of the strongest absorption line under consideration, in our case the $\nu_{2}$ $3_{1,2}-3_{0,3}$ transition. However, the perceived astrophysical absorption from this transition is greatly affected by its telluric counterpart, and the line depth is highly dependent on the atmospheric division procedure. The same is true for the next strongest line, the $\nu_{2} 1_{1,1}-0_{0,0}$ transition. We use both lines in estimating the minimum covering fraction, choosing $f_{c}=0.25$, but caution that this is highly uncertain. Fits using $f_{c}=0.25$ are shown as blue curves in Figure 2, and resulting parameters are again in Table 1. Inferred column densities are plotted as open blue diamonds in Figure 3. Scaling by the covering fraction demonstrates that column densities inferred from optically thick transitions increase much more than those inferred from optically thin transitions as $f_{c}$ decreases, but at $f_{c}=0.25$ the saturated $\nu_{2} 3_{1,2}-3_{0,3}$ and $\nu_{2} 1_{1,1}-0_{0,0}$ transitions only provide lower limits on column densities. A fit to the seven unsaturated transitions is shown by the blue dash-dot line in Figure 3 and corresponds to $N\left(\mathrm{H}_{2} \mathrm{O}\right)=(1.3 \pm 0.3) \times 10^{19} \mathrm{~cm}^{-2}$ in LTE at $T=640 \pm 80 \mathrm{~K}$.

\section{DISCUSSION}

The pure rotational $1_{1,1}-0_{0,0}$ transition of $\mathrm{H}_{2} \mathrm{O}$ at $1113.343 \mathrm{GHz}(269.3 \mu \mathrm{m})$ is seen in both emission and absorption toward AFGL 2591 (van der Tak et al. 2013; Kaźmierczak-Barthel et al. 2014; Choi et al. 2015). The emission component arises in the protostellar envelope, and the absorption components in the blueshifted outflow and foreground gas. This absorption probes the same quantum state as the $\nu_{2} 1_{1,1}-0_{0,0}$ transition, but a direct comparison is hindered by several effects. The emitting regions at $6 \mu \mathrm{m}$ and $1.1 \mathrm{THz}(\sim 270 \mu \mathrm{m})$ are likely different, so that gas probed by one transition may not necessarily be probed by the other. Our EXES observations used a 9."9 by 1.9 slit centered on VLA 3, while HIFI observations at $1113 \mathrm{GHz}$ have a roughly circular beam with FWHM $\sim 19^{\prime \prime}$, again meaning that different regions are being probed. Finally, emission from the $\left(v_{1} v_{2} v_{3}\right) J_{K_{a}, K_{c}}=(000) 1_{1,1}$ state $(53.4 \mathrm{~K}$ above ground) is extremely strong and interferes with the absorption features at $1113 \mathrm{GHz}$, whereas emission from the $(010) 1_{1,1}$ state $(2352 \mathrm{~K}$ above ground) is not observed, leaving the $6.1163311 \mu \mathrm{m}$ line unobscured. All of these effects must be considered when comparing any results from HIFI and EXES.

As described in Section 1, different components of AFGL 2591 are distinguished by line of sight velocities, line profiles, and observed molecules. The $\mathrm{H}_{2} \mathrm{O}$ absorption lines are centered at roughly $-16 \mathrm{~km} \mathrm{~s}^{-1}$ with FWHM $\sim 20 \mathrm{~km} \mathrm{~s}^{-1}$ for the three lowest-lying levels and shift to $-11 \mathrm{~km} \mathrm{~s}^{-1}$ with FWHM $\sim 13 \mathrm{~km} \mathrm{~s}^{-1}$ for the higher-lying levels, best matching the blueshifted outflow. These line profiles are most similar to absorption seen in $\mathrm{HCN}$ and $\mathrm{C}_{2} \mathrm{H}_{2}$ (Knez 2006, observed at $13 \mu \mathrm{m})$ as well as specific absorption components of ${ }^{13} \mathrm{CO}$ and vibrationally excited ${ }^{12} \mathrm{CO}$ (Mitchell et al. 1989; van der Tak et al. 1999, observed at $4.7 \mu \mathrm{m}$ ), all of which are thought to arise in hot, dense gas close to the central protostar. Several $22 \mathrm{GHz} \mathrm{H}_{2} \mathrm{O}$ masers are observed throughout the region, with many concentrated in the walls of the blueshifted outflow associated with VLA 3 (Trinidad et al. 2003; Sanna et al. 2012; Torrelles et al. 2014). Although maser velocities are primarily at $v_{\mathrm{LSR}} \leqslant-18 \mathrm{~km} \mathrm{~s}^{-1}$, absorption by gas in small, shocked clumps could explain the small covering fraction required by our analysis. Given the above, we posit that the $\mathrm{H}_{2} \mathrm{O}$ absorption observed with EXES arises in hot, dense gas at the base of the blueshifted outflow.

Water emission attributed to the outflow component is observed with HIFI in multiple transitions as a broad feature (van der Tak et al. 2013; Kaźmierczak-Barthel et al. 2014; Choi et al. 2015), but is centered closer to the systemic velocity of the envelope than the absorption we see. Analysis of the $\mathrm{H}_{2} \mathrm{O}$ outflow emission by Choi et al. (2015) indicates a temperature of $T \sim 70-90 \mathrm{~K}$ and column density of $N\left(\mathrm{H}_{2} \mathrm{O}\right) \sim 4 \times 10^{13} \mathrm{~cm}^{-2}$, similar to results found by Karska et al. (2014) who analyzed unresolved $\mathrm{H}_{2} \mathrm{O}$ absorption observed with PACS on Herschel, finding $T=160 \pm 130 \mathrm{~K}$ and $N\left(\mathrm{H}_{2} \mathrm{O}\right) \sim 10^{14} \mathrm{~cm}^{-2}$. These values are significantly below what we find, suggesting that EXES and HIFI/PACS observations trace different components. Between the broad and narrow water emission observed with HIFI, the broad absorption observed with EXES, and the abundant maser spots, it is evident that the water-containing gas in AFGL 2591 is both spatially and kinematically complex. Interpreting the various observations of $\mathrm{H}_{2} \mathrm{O}$ in AFGL 2591 in unison will require utilizing a physical model that includes radiative transfer accounting for absorption, stimulated and spontaneous emission, and collisional (de-)excitation, as well as kinematic and geometric effects.

\section{SUMMARY}

We have detected ten absorption features arising from warm gas in AFGL 2591 caused by ro-vibrational transitions of water, including seven from the $\nu_{2}$ band of $\mathrm{H}_{2} \mathrm{O}$, two from the vibrationally excited $\nu_{2}$ band of $\mathrm{H}_{2} \mathrm{O}$, and one from the $\nu_{2}$ band of $\mathrm{H}_{2}^{18} \mathrm{O}$. Among the detected absorption lines is the $\nu_{2} 1_{1,1}-0_{0,0}$ transition at $6.1163311 \mu \mathrm{m}$, which probes the ground state of para $-\mathrm{H}_{2} \mathrm{O}$. Relative strengths of absorption features are suggestive of a covering fraction less than 1 (or absorption arising within the $6 \mu \mathrm{m}$ emitting photosphere), with a limit of $f_{c} \geqslant 0.25$ set by the depth of the strongest absorption features. Analysis of the level populations assuming $f_{c}=0.25$ results in $N\left(\mathrm{H}_{2} \mathrm{O}\right)=(1.3 \pm 0.3) \times 10^{19} \mathrm{~cm}^{-2}$ for LTE at $T=640 \pm 80 \mathrm{~K}$. Line profiles best match the blueshifted outflow component, and we ascribe the absorption to hot, dense gas at the base of the outflow. The temperature and column density inferred by our analysis are much larger than those reported by Choi et al. (2015) for the outflow component observed in $\mathrm{H}_{2} \mathrm{O}$ emission by HIFI. Uncertainty in whether the EXES and HIFI observations are probing the same gas makes the combined interpretation of both datasets difficult, and a physical model of AFGL 2591 that includes radiative transfer will be necessary for such an analysis. Clearly though, observations of the $\nu_{2}$ ro-vibrational band of $\mathrm{H}_{2} \mathrm{O}$ at high spectral resolution-observations uniquely achievable with EXES on SOFIA - add important information for interpreting this region and others.

M. J. R. and C. N. D. acknowledge Collaborative Agreement NNX13AI85A between UCD and NASA Ames for its support and support of EXES development. Many thanks to the anonymous referee.

\section{REFERENCES}

Boonman, A. M. S., \& van Dishoeck, E. F. 2003, A\&A, 403, 1003 Carr, J. S., Tokunaga, A. T., \& Najita, J. 2004, ApJ, 603, 213 
Cheung, A. C., Rank, D. M., Townes, C. H., Thornton, D. D., \& Welch, W. J. 1969, Natur, 221, 626

Choi, Y., van der Tak, F. F. S., van Dishoeck, E. F., Herpin, F., \& Wyrowski, F. 2015, A\&A, in press (arXiv:1412.4818)

Clarke, M., Vacca, W. D., \& Shuping, R. Y. 2015, in ASP Conf. Ser. 24, Astronomical Data Analysis Software and Systems XXIV, ed. A. R. Taylor \& J. M. Stil (San Francisco, CA: ASP), in press

Cushing, M. C., Vacca, W. D., \& Rayner, J. T. 2004, PASP, 116, 362

de Graauw, T., Haser, L. N., Beintema, D. A., et al. 1996, A\&A, 315, L49

de Graauw, T., Helmich, F. P., Phillips, T. G., et al. 2010, A\&A, 518, L6

Emprechtinger, M., Monje, R. R., van der Tak, F. F. S., et al. 2012, ApJS, 756,136

Flagey, N., Goldsmith, P. F., Lis, D. C., et al. 2013, ApJ, 762, 11

Indriolo, N., Neufeld, D. A., Seifahrt, A., \& Richter, M. J. 2013, ApJ, 776, 8 Johnston, K. G., Shepherd, D. S., Robitaille, T. P., \& Wood, K. 2013, A\&A, 551, A43

Karska, A., Herpin, F., Bruderer, S., et al. 2014, A\&A, 562, A45

Kaźmierczak-Barthel, M., van der Tak, F. F. S., Helmich, F. P., et al. 2014, A\&A, 567, A53

Kessler, M. F., Steinz, J. A., Anderegg, M. E., et al. 1996, A\&A, 315, L27

Knez, C. 2006, PhD thesis, Univ. Texas

Lacy, J. H., Richter, M. J., Greathouse, T. K., Jaffe, D. T., \& Zhu, Q. 2002, PASP, 114, 153

Lada, C. J., Thronson, H. A., Jr., Smith, H. A., Schwartz, P. R., \& Glaccum, W. 1984, ApJ, 286, 302

Melnick, G. J., Ashby, M. L. N., Plume, R., et al. 2000, ApJL, 539, L87

Melnick, G. J., Stauffer, J. R., Ashby, M. L. N., et al. 2000, ApJL, 539, L77

Mitchell, G. F., Curry, C., Maillard, J.-P., \& Allen, M. 1989, ApJ, 341, 1020
Najita, J. R., Edwards, S., Basri, G., \& Carr, J. 2000, Protostars and Planets IV (Tucson, AZ: Univ. Arizona Press)

Pilbratt, G. L., Riedinger, J. R., Passvogel, T., et al. 2010, A\&A, 518, L1

Pontoppidan, K. M., Salyk, C., Blake, G. A., \& Käufl, H. U. 2010, ApJL, 722, L173

Richter, M. J., Ennico, K. A., McKelvey, M. E., \& Seifahrt, A. 2010, Proc. SPIE, 7735, 77356Q

Rothman, L. S., Gordon, I. E., Babikov, Y., et al. 2013, JQSRT, 130, 4

Salyk, C., Pontoppidan, K. M., Blake, G. A., et al. 2008, ApJL, 676, L49

Sanna, A., Reid, M. J., Carrasco-González, C., et al. 2012, ApJ, 745, 191

Snell, R. L., Howe, J. E., Ashby, M. L. N., et al. 2000, ApJL, 539, L101

Sonnentrucker, P., Neufeld, D. A., Phillips, T. G., et al. 2010, A\&A, 521, L12

Torrelles, J. M., Trinidad, M. A., Curiel, S., et al. 2014, MNRAS, 437, 3803

Trinidad, M. A., Curiel, S., Cantó, J., et al. 2003, ApJ, 589, 386

van der Tak, F. F. S., van Dishoeck, E. F., Evans, N. J., II, Bakker, E. J., \& Blake, G. A. 1999, ApJ, 522, 991

van der Tak, F. F. S., Walmsley, C. M., Herpin, F., \& Ceccarelli, C. 2006, A\&A, 447, 1011

van der Tak, F. F. S., Chavarría, L., Herpin, F., et al. 2013, A\&A, 554, A83

van der Wiel, M. H. D., van der Tak, F. F. S., Spaans, M., et al. 2011, A\&A, 532, A88

van Dishoeck, E. F., Herbst, E., \& Neufeld, D. A. 2013, ChRv, 113, 9043

van Dishoeck, E. F., Kristensen, L. E., Benz, A. O., et al. 2011, PASP, 123,138

Wang, K.-S., van der Tak, F. F. S., \& Hogerheijde, M. R. 2012, A\&A, 543, A22

Young, E. T., Becklin, E. E., Marcum, P. M., et al. 2012, ApJL, 749, L17 